\title{
Efficacy of Immune Checkpoint Inhibitors in Metastatic Epstein-Barr Virus Associated Gastric Cancer (EBVaGC): A Case Series and Review of Literature
}

\author{
Shimeng Liang \\ West China Hospital of Sichuan University \\ Weibing Leng \\ West China Hospital of Sichuan University \\ Dan Jiang \\ West China Hospital of Sichuan University \\ Ming Liu \\ West China Hospital of Sichuan University \\ Dan Cao \\ West China Hospital of Sichuan University \\ Qing Zhu \\ West China Hospital of Sichuan University \\ Yongsheng Wang \\ West China Hospital of Sichuan University \\ Meng Qiu ( $\square$ qiumeng33@hotmail.com ) \\ West China Hospital of Sichuan University
}

\section{Research Article}

Keywords: metastatic gastric cancer, epstein-barr virus-associated gastric carcinoma, immunotherapy, immune checkpoint inhibitors, programmed cell death protein 1 inhibitors

Posted Date: November 30th, 2021

DOI: https://doi.org/10.21203/rs.3.rs-967167/v1

License: () (7) This work is licensed under a Creative Commons Attribution 4.0 International License. Read Full License 


\section{Abstract \\ Background}

Immunotherapy has revolutionized the treatment of malignant tumors. However, limited clinical data are available to report the efficacy of immune checkpoint inhibitors (ICls) on Epstein-Barr virus-associated gastric carcinoma.

\section{Methods}

In this study, we report a case series of five patients with metastatic Epstein-Barr virus-associated gastric carcinoma who were treated with ICls and to perform a pooled analysis of published cases to investigate the efficacy of ICls in Epstein-Barr virus-associated gastric carcinoma patients.

\section{Results}

Between 2018 and 2020, five metastatic gastric cancer patients with EBV positivity who received PD-1 antibodies treatment were included in the analysis at the authors' institution. Furthermore, we performed a pooled analysis of the contemporary literature. In our case series, two patients experienced partial response (PR); one patient achieved complete response (CR), whereas two patients had progression disease (PD), resulting an ORR of $60 \%$. In the pooled analysis of all 36 patients, ORR was $48.6 \%$ (17/35). For the first line and later lines, it was $75 \%(3 / 4)$ and $45.2 \%$ (14/31) respectively. The ORR was $46.7 \%$ $(14 / 30)$ for ICls monotherapy and improved to $60 \%(3 / 5)$ by combination with chemotherapy.

\section{Conclusions}

These results demonstrated that an EBV-positive status was a reliable biomarker for immunotherapy in metastatic gastric cancer, especially for monotherapy. Immunotherapy combined with chemotherapy may be a better strategy, warranting further large-scale clinical trials for validation.

\section{Background}

According to the Global Cancer Statistics 2018, gastric cancer (GC) is the fifth most frequently diagnosed cancer and the third leading cause of cancer death (1). Advanced gastric cancer patients have a poor prognosis with a median survival time, if treated with chemotherapy, of less than one year. Checkpoint inhibitor-based immunotherapy for gastric cancer has yielded promising overall survival (OS) in recent years. Anti-PD-1 antibody has been approved for the third-line treatment of advanced gastric cancer according to the results of KEYNOTE-059 and ATTRACTION-2 trials $(2,3)$. However, the ORR of PD-1 inhibitors monotherapy was only about $10 \%$ in above-mentioned clinical trials. Pembrolizumab in KEYNOTE-061 didn't show significant improvement in OS compared with paclitaxel as second-line therapy (4). Checkmate 649 and ATTRACTION-4 both demonstrated that anti-PD-1 antibody plus chemotherapy was superior to chemotherapy in PFS or OS $(5,6)$ while KEYNOTE-062 didn't (7). The low effective rate of immunotherapy alone and controversial survival outcomes in these trials underline the urgent need to identify biomarkers for patient selection to increase the clinical benefit from immune checkpoint inhibitors (ICls) in GC.

By analysis of TCGA data, EBVaGC exhibits strong evidence of immune infiltration, higher lymphocytic infiltration and higher expression of immune checkpoint pathway genes(8), indicating that EBV-positive status was a potential biomarker for immunotherapy in gastric cancer (9). Kim et al reported that EBVaGC patients administrated with pembrolizumab after first-line therapy all reached PR (10). However, other studies showed the ORR was only $16.7 \%-28.6 \%$ for EBVaGC patients received anti-PD-1 antibody alone after first-line therapy (11-14), which was only comparable with or slightly higher than the response rate of pembrolizumab alone in total population of KEYNOTE-059 and KEYNOTE-061 $(3,4)$. This contradictory phenomenon highlights the significance to explore whether EBV positivity is an effective response predictor of immune checkpoint inhibitors (ICls) and which regimen is optimal for EBVaGC. In view of limited evaluation on the efficacy of ICls on EBVaGC, we reported a case series of five patients with metastatic EBVaGC who received ICls treatment in our hospital and performed a pooled analysis of published cases to investigate the role of EBV-positive status in prediction of immunotherapy efficacy.

\section{Methods}

From May 2018 to 2020, we collected metastatic EBVaGC patients received PD-1 antibodies treatment in the Departments of Abdominal Oncology of West China Hospital. RECIST 1.1 criterion was used to evaluate responses to treatment. The eligibility criteria were the presence of histologically proven EBVaGC and availability of response to treatment data and clinicopathological characteristics. All participating patients were at TNM staging IV and received at least one cycle of ICls. Patients were treated with ICls alone or combined with chemotherapy. For estimation of ORR, the best responses in the case report or case series were used.

A systematic search in Google Scholar and PubMed databases was carried out using the following key words: immunotherapy, immune checkpoint inhibitors, PD-1, PD-L1, anti-PD-1 therapy, anti-PD-L1 therapy, Epstein-Barr virus-associated gastric carcinoma, EBVaGC, Epstein-Barr virus positive, Epstein-Barr virus, metastatic gastric cancer.

\section{Results}

\section{Case Series: The Clinicopathologic Characteristics}


From May 2018 to Dec 2020, five metastatic EBVaGC patients received PD-1 antibodies treatment in the Departments of Abdominal Oncology of West China Hospital. The clinicopathologic and genetic characteristics of the five patients are summarized in Table 1. The median age was 47.2 years (range, 27-59y) and most patients were male (4/5). All patients were adenocarcinoma, and four morphologically characterized as lymphoepithelioma-like carcinoma and shows increased lymphocyte infiltration in the tumor microenvironment. EBV status was focally positive for EBV-encoded small RNA (EBER) in the tumor cells by in situ hybridization (ISH) on unstained slides from paraffin-embedded tumor blocks. Moreover, three patients performed next generation sequencing (NGS) test, and the results including genomic alterations, PD-L1 expression and tumor mutation burden (TMB) were listed in Table 1. The tissue was mismatch repair proficient (pMMR) or microsatellite stable (MSS) in every patient if detected. As for personal history and family history, two patients had a smoking history of more than 20 years and a patient had a sister who was diagnosed with EBV+ NK/T cell lymphoma.

\section{Case Series: The Treatment Information}

The detailed treatment information was also listed in Table 1. Only one patient received curative gastrectomy and then developed recurrence and metastasis four years later. Rest of them initially lacked the chance of radical surgery at baseline and only got a palliative resection or exploratory operation. ICls alone or combined with chemotherapy were used as the first-line, second-line, and third-line in 2, 2 and one patient(s) respectively. Before immunotherapy, one patient with peritoneal metastases received systemic SOX (S-1 combined with oxalipaltin) plus intraperitoneal paclitaxel chemotherapy and one received SOX as first line treatment; one experienced a progression after SOX then TP chemotherapy. Involved immunotherapy drugs included Pembrolizumab and Sintilimab (PD-1 antibody approved by China Food and Drug Administration). Three patients were administrated immunotherapy in combination with chemotherapy and two patients had monotherapy. One patient was treated with Sintilimab plus Paclitaxel-albumin, one was Sintilimab plus SOX, and one was Pembrolizumab plus PF (Cisplatin, Fluorouracil). Among all patients, two achieved partial response (PR), and one even obtained complete response (CR), leading an ORR and rate of disease control (DCR) of $60 \%$ (Fig. 1). Two untreated patients both achieved PR with an ORR of $100 \%(2 / 2)$ and the ORR for later-line was $33.3 \%$ (1/3). The mean PFS was 5.2 months (range from 1.2 months to not reach) and two patients were still in response. One patient (No.3) achieved CR after seven doses of Sintilimab as the second-line treatment and so far with the duration of response exceeding ten months. CR status was further proved by endoscopic biopsy and PET/CT. Two patients (No.2 \& No.4) had disease progressed after 2 cycles of ICls and died in a short time, and one of them even had hyperprogression. No patients had severe immune toxicity. The mean number of cycles of immunotherapy was 5.6 (ranging from 2 to 16).

Table 1

The Clinicopathologic, Gene Characteristics and Treatment Features of Patients in Our Institution.

\begin{tabular}{|c|c|c|c|c|c|c|c|c|c|c|c|c|}
\hline No. & Age & Sex & $\begin{array}{l}\text { ECOG } \\
\text { PS } \\
\text { Score }\end{array}$ & Pathology & $\begin{array}{l}\text { Primary } \\
\text { lesion }\end{array}$ & Metastases & $\begin{array}{l}\text { Previous } \\
\text { regimens }\end{array}$ & Immunotherapy & MMR & MSI & HER2 & PD-L1 \\
\hline \multirow[t]{2}{*}{1} & 45 & M & 0 & $\begin{array}{l}\text { Partly LE-like } \\
\text { ca }\end{array}$ & body & $\begin{array}{l}\text { Lymph } \\
\text { nodes }\end{array}$ & $\begin{array}{l}\text { Radical } \\
\text { Surgery }\end{array}$ & Sintilimab* $4+S O X \star 5$ & NA & MSS & 0 & $\begin{array}{l}\text { TPS }=30 \% \\
\text { CPS }=5\end{array}$ \\
\hline & & & & & & liver & & & & & & \\
\hline \multirow[t]{2}{*}{2} & 54 & M & 1 & $\begin{array}{l}\text { poorly } \\
\text { differentiated } \\
\text { AG }\end{array}$ & $\begin{array}{l}\text { Body- } \\
\text { fundus } \\
\text { coniunction }\end{array}$ & $\begin{array}{l}\text { Lymph } \\
\text { nodes }\end{array}$ & sox+Tip & $\begin{array}{l}\text { Sintilimab+Paclitaxel- } \\
\text { albumin*2 }\end{array}$ & pMMR & MSS & 0 & $\begin{array}{l}\text { TPS }<1 \% \\
\text { CPS }<1\end{array}$ \\
\hline & & & & & & Peritoneum & & & & & & \\
\hline 3 & 59 & M & 0 & LE-like ca & body & Peritoneum & sox & Sintilimab*25 & NA & NA & 0 & NA \\
\hline 4 & 27 & $\mathrm{~F}$ & 1 & LE-like ca & body & $\begin{array}{l}\text { Peritoneum } \\
\text { Liver }\end{array}$ & $\mathrm{SOX} \rightarrow \mathrm{TP}$ & Pembrolizumab*2 & pMMR & MSS & 0 & $\begin{array}{l}\text { TPS }=5 \% \\
\text { CPS }=6\end{array}$ \\
\hline 5 & 51 & $M$ & 0 & LE-like ca & Body & $\begin{array}{l}\text { Lymph } \\
\text { nodes liver }\end{array}$ & NA & $\begin{array}{l}\text { Pembrolizumab+ } \\
\mathrm{PF}^{\star} 4\end{array}$ & pMMR & NA & 0 & NA \\
\hline
\end{tabular}

$M$ indicates male; $F$, female; NA, no available; PD-1, programmed death 1; PD-L1, programmed cell death protein 1; AG, adenocarcinoma gastric cancer; SRC, $s$ $\mathrm{N}$, no; ca, carcinoma; PR, partial response; SD, stable disease; PD, progression disease; SOX, S1+ oxaliplatin; XELOX, capecitabine + oxaliplatin; PF, cisplatin+ 1 intraperitoneal paclitaxel; pMMR, proficient DNA mismatch repair; MSS, microsatellite instability-stable; TMB, tumor burden; PFS, progression-free survival; O؟ no progression at the last follow-up.

\section{Review Results}

As of 2020/12/31, 36 cases (including the present five cases) from 6 reports were found eligible for the present analysis (9-14). Patients were pathologically confirmed EBVaGC and from four countries including Canada, China, Japan and Korea. The references, patient numbers, pathology, PD-L1 expression and treatment were listed in Table 2. All patients were MSS or pMMR if the tissue was detected for these items, so these results were not included in the table. Except for 11 patients whose PD-L1 ex-pression were no report, majority of them were positive for it with 19 patients whose CPS $>1$. PD-1 inhibitors including nivolumab, pembrolizumab, camrelizumab, toripalimab and sintilimab were used as monotherapy or combined with chemotherapy or other ICls. Monotherapy shared the most percentage of $86.1 \%$ and ICls plus chemo-therapy were used in only five patients. $88.9 \%$ of patients had a history of anti-tumor therapy while only four patients received ICls as first line. Due to limited information, we only summarized the ORR for survival analysis. Among all patients, the ORR was $48.6 \%$ with 16 patients experience PR and one CR. For monotherapy, the ORR was $46.7 \%$ and for combination with chemotherapy was $60 \%$. The ORR was $75 \%$ for the first-line and $45.2 \%$ for the later-lines respectively. 
Table 2

The Summary of Response to ICls in Metastatic EBVaGC in Published Papers.

\begin{tabular}{|c|c|c|c|c|c|c|}
\hline References & $\begin{array}{l}\text { Num of } \\
\text { Pts }\end{array}$ & Pathology & PD-L1 & Immunotherapy & Previous lines & Response \\
\hline (Panda, A., et al. 2018) & 1 & AG & NR & Avelumab & 2 & PR \\
\hline \multirow[t]{6}{*}{ (Kim, S.T., et al.2018) } & \multirow[t]{6}{*}{6} & \multirow[t]{6}{*}{ AG } & CPS:5 & \multirow[t]{6}{*}{ Pembrolizumab } & \multirow[t]{6}{*}{$>=1$} & \multirow[t]{6}{*}{ PR } \\
\hline & & & CPS:1 & & & \\
\hline & & & CPS:80 & & & \\
\hline & & & CPS:80 & & & \\
\hline & & & CPS:35 & & & \\
\hline & & & CPS:15 & & & \\
\hline \multirow{4}{*}{$\begin{array}{l}\text { (Mishima, S., et al. } \\
\text { 2019) }\end{array}$} & \multirow[t]{4}{*}{4} & \multirow[t]{4}{*}{ AG } & \multirow[t]{4}{*}{$\mathrm{CPS}>=1$} & \multirow[t]{4}{*}{ Nivolumab } & \multirow[t]{4}{*}{$>=2$} & PR \\
\hline & & & & & & $N R^{\star 3}$ \\
\hline & & & & & & $N R^{\star 3}$ \\
\hline & & & & & & $N R^{\star 3}$ \\
\hline \multirow[t]{9}{*}{ (Xie, T., et al.2020) } & \multirow[t]{9}{*}{9} & AG & CPS:5 & PD-1 inhibitor* & 1 & SD \\
\hline & & \multirow[t]{8}{*}{ SRC } & CPS:10 & PD-L1 inhibitor*1 & 2 & PR \\
\hline & & & CPS:5 & PD-1 inhibitor* + XELOX & 1 & SD \\
\hline & & & CPS:0 & Nivolumab+ipilimumab $\rightarrow$ nivolumab & 2 & SD \\
\hline & & & CPS:30 & Nivolumab $\rightarrow$ nivolumab+S1 & 2 & SD \\
\hline & & & CPS:20 & Nivolumab & 0 & SD \\
\hline & & & CPS:50 & & 0 & PR \\
\hline & & & CPS: 10 & & 1 & PR \\
\hline & & & CPS: 0 & & 1 & $\star^{2}$ \\
\hline \multirow[t]{7}{*}{ (Qiu, M.-Z., et al.2020) } & \multirow[t]{7}{*}{7} & \multirow[t]{7}{*}{ AG } & \multirow[t]{7}{*}{ NR } & 4 camrelizumab & \multirow{7}{*}{$\begin{array}{l}2 \text { pts had } 1 \text { line; } 5 p t s \text { had } 2 \\
\text { lines }\end{array}$} & PR \\
\hline & & & & \multirow[t]{6}{*}{3 toripalimab } & & PR \\
\hline & & & & & & SD \\
\hline & & & & & & SD \\
\hline & & & & & & PD \\
\hline & & & & & & PD \\
\hline & & & & & & PD \\
\hline Our institution & 5 & $A G$ & TPS $=30 \%, C P S=5$ & Sintilimab+SOX & 0 & PR \\
\hline \multirow[t]{4}{*}{ Our institution } & \multirow[t]{4}{*}{5} & \multirow[t]{4}{*}{ AG } & TPS $<1 \%$, CPS $<1$ & Sintilimab+Paclitaxel-albumin & 1 & PD \\
\hline & & & NA & Sintilimab & 1 & $\mathrm{CR}$ \\
\hline & & & TPS=5\%, CPS=6 & pembrolizumab & 2 & PD \\
\hline & & & NA & pembrolizumab+PF & 0 & PR \\
\hline
\end{tabular}

\section{Discussion}

EBVaGC is one of the four major genomic gastric cancer types defined by The Cancer Genome Atlas (TCGA) in 2014, associated with $8.7 \%$ of gastric carcinoma worldwide (8). Compared with EBV-non associated GC (EBVnGC), EBVaGC is characterized by recurrent PIK3CA mutations, extreme DNA hypermethylation, and amplification of JAK2, CD274 (also known as PD-L1) and PDCD1LG2 (also known as PD-L2) according to TCGA subtype. Usually, patients with EBVaGC present a best prognosis for both recurrence-free survival and overall survival, and they seemed to be chemotherapy-resistant and the ORR for first-line chemotherapy were $29.0 \%$, nearly half of that of EBV-negative GC(15). Extensive lymphocyte infiltration and constitutive expression of immunosuppressive molecules PD-L1/PD-L2 in EBVaGC highlight the potential of EBV status as a novel biomarker for immunotherapy efficacy in gastric cancer. In 2018, Kim et al conducted a single-center, phase 2 trial in which 61 unselected patients with metastatic GC received pembrolizumab monotherapy 
and performed integrated molecular profiling(10). Dramatic responses of EBVaGC to pembrolizumab were observed with an ORR of $100 \%$, but the study only included six patients. In contrast, another four studies showed the ORR was only $16.7 \%-28.6 \%$ for EBVaGC patients received anti-PD- 1 antibody alone after first-line therapy $(11-14)$. In this article, we reported the efficacy of ICls in a case series of metastatic EBVaGC patients in our institute and then performed a pooled analysis. The ORR was $60 \%$ for the five cases in our hospital, while $48.6 \%$ for all patients in the pooled analysis. Moreover, based on the results of pooled analysis, the ORR of ICls monotherapy on EBVaGC was better than that of ICls monotherapy on unselected GC in ATTRACTION-2 trial (46.7\% vs $11.9 \%$ ) 3. These results demonstrated that an EBV positive status was a reliable biomarker for immunotherapy in metastatic gastric cancer. ICls combined with chemotherapy seemed to have a better response than immunotherapy alone with an ORR of $60 \%$ and $46.7 \%$ respectively, indicating immunotherapy combined with chemotherapy maybe a better strategy than immunotherapy alone for the first line treatment of metastatic EBVaGC.

Besides EBV status, other potential biomarkers of ICls used in clinical practice include MSI, TMB, PD-L1(3, 12, 16-19). Microsatellite instability (MSI) status and tumor mutation burden (TMB) have been widely accepted as potential biomarkers for response to anti-PD-1/PD-L1 antibody in many types of cancer. PDL1 expression was also usually associated with good response rate for immunotherapy in gastric cancer. However, these biomarkers could not completely cover EBV positive gastric cancer, which also benefits from immunotherapy. First, MSI-H is mutually exclusive with EBV positivity (10). Second, majority of EBVaGC are TMB-L (20), such as the patient No.1 in our case series. Third, Although EBVaGC is characterized by marked high expression of PD-L1(12), a substantial proportion of it does not express high levels of PD-L1(14). Moreover, the relationship between PD-L1 expression and efficacy of ICls was inconsistent across different trials (21), and even PD-L1 positive patients were possible to resistant to immunotherapy. In short, TMB-H, MSI-H, PD-L1 or EBV positive patients would be more likely to benefit from immunotherapy. With the current understanding of cancer immunology, a single parameter may not be sufficient to predict immunotherapy efficacy. Therefore, the future research of biomarker should address the integration of multi-omics biomarkers with an interdisciplinary approach.

It is accepted that ICls require an endogenous adaptive immune response to be effective and EBVaGC is considered beneficial owing to presumably activated T cells in response to viral antigens (22). However, large heterogeneity exists in the immune contexture of GC even in its subtype; favorable factors and downsides were found in types traditionally insensitive and sensitive to ICls (23). This kind of heterogeneity partly explains why patients displayed diverse response to ICls in our cases and it is reasonable to relate efficacy of immunotherapy to tumor immune environment (TME) (24). Researchers proposed a way of categorizing patients under four TME groups with potential implications for mechanism and therapy according to PD-L1 expression and the presence of TILs in tumor biopsies (25), (26). About $65 \%$ of EBVaGC cases fell into type I (TILs+ PD-L1+) or type IV (TILs+ PD-L1-) microenvironments (27). In our cases, genetic testing of patient No.2 and No.4 were consistent with this finding since they were type IV and I respectively. Overregulation of activated TILs due to the effect of the B7-H1/PD-1 pathway and potentially other T cell regulatory pathways, dysfunctional TILs in the TME due to suppression by molecular pathways may explain the antitumor immune defect in EBVaGC patients (28). More basic and clinical researches are in need of exploration.

Ongoing clinical trials about ICls efficacy in GC including EBV positive subtypes are all enumerated in the Table 3. They are phase II or I/II which objective population are not limited to advanced patients. PD-1/PD-L1 antibody plus chemotherapy is the main condition instead of monotherapy; some drugs are designed in combination with CTLA-4 inhibitors or antiangiogenic therapy. We are convinced that with more prospective large-scale clinical tests, a better standard treatment for EBVaGC would be finally found.

Table 3

Ongoing Trials Evaluating Immune Checkpoint Inhibitors efficacy related to EBVaGC

\begin{tabular}{|c|c|c|c|c|c|c|}
\hline $\begin{array}{l}\text { Study } \\
\text { identifier }\end{array}$ & Status & Stage & Drug treatment & Outcome Measures & Phases & $\begin{array}{l}\text { Study } \\
\text { Designs }\end{array}$ \\
\hline NCT04675866 & $\begin{array}{l}\text { Not yet } \\
\text { recruiting }\end{array}$ & Advanced & $\begin{array}{l}\text { Camrelizumab+S-1+Albumin-bound } \\
\text { paclitaxel }\end{array}$ & ORR/DCR/OS/PFS & II & $\begin{array}{l}\text { Single Group } \\
\text { Assignment }\end{array}$ \\
\hline NCT04202601 & Recruiting & Advanced & Sintilimab+|BI310(CTLA-4 antibody) & $\mathrm{pCR} / \mathrm{ORR}$ & $\mathrm{I} / \mathrm{II}$ & $\begin{array}{l}\text { Single Group } \\
\text { Assignment }\end{array}$ \\
\hline NCT04152889 & Recruiting & III & Camrelizumab+S-1+Docetaxel & DFS & II & $\begin{array}{l}\text { Single Group } \\
\text { Assignment }\end{array}$ \\
\hline NCT03755440 & $\begin{array}{l}\text { Enrolling by } \\
\text { invitation }\end{array}$ & Metastatic & SHR-1210(PD-1 antibody) & RR/PFS/OS/DCR & II & $\begin{array}{l}\text { Single Group } \\
\text { Assignment }\end{array}$ \\
\hline NCT03257163 & Recruiting & IB-IIIC & Capecitabine, Pembrolizumab & RFS rate & II & $\begin{array}{l}\text { Single Group } \\
\text { Assignment }\end{array}$ \\
\hline NCT04250948 & Recruiting & $\begin{array}{l}\text { Locally } \\
\text { Advanced }\end{array}$ & $\begin{array}{l}\text { JS001(PD-L1 antibody) } \\
\text { +Oxaliplatin+S1+Capecitabine }\end{array}$ & $\begin{array}{l}\text { TRG0/1/pCR/R0 resection rate } \\
\text { /RFS/ORR/DCR/OS }\end{array}$ & II & $\begin{array}{l}\text { Parallel } \\
\text { Assignment }\end{array}$ \\
\hline NCT04632459 & $\begin{array}{l}\text { Not yet } \\
\text { recruiting }\end{array}$ & Metastatic & Pembrolizumab +ramucirumab & ORR & II & $\begin{array}{l}\text { Single Group } \\
\text { Assignment }\end{array}$ \\
\hline NCT03751761 & Recruiting & Metastatic & $\begin{array}{l}\text { durvalumab/tremelimumab (CTLA-4 } \\
\text { antibody) + paclitaxel }\end{array}$ & $\mathrm{RR}$ & $\mathrm{I} / \mathrm{II}$ & $\begin{array}{l}\text { Single Group } \\
\text { Assignment }\end{array}$ \\
\hline КСТ0004186 & Recruiting & Advanced & paclitaxel+nivolumab & MTD; RP2D; PFS & $\mathrm{lb} / \mathrm{II}$ & $\begin{array}{l}\text { Single Group, } \\
\text { Blinding }\end{array}$ \\
\hline
\end{tabular}

RFS, recurrence-free survival; MTD, maximal tolerated dose; RP2D, recommended phase 2 dose; GEJ, gastroesophageal junction; pCR, pathologic complete response

\section{Conclusions}


To sum up, an EBV-positive status is a reliable biomarker for immunotherapy in metastatic gastric cancer. Prospective clinical trials are needed to validate the efficacy of immunotherapy in combination with chemotherapy.

\section{List Of Abbreviations}

ICls, immune checkpoint inhibitors; EBV, Epstein-Barr virus; EBVaGC, Epstein-Barr virus-associated gastric carcinoma; PR, partial response; CR, complete response; PD, progression disease, ORR, Objective response rate; GC, gastric cancer; OS, overall survival; PFS, progression-free survival; EBER, EBV-encoded small RNA; ISH, in situ hybridization; NGS, next generation sequencing; TMB, tumor mutation burden; pMMR, mismatch repair proficient; MSS, microsatellite stable; SOX, S-1 + oxaliplatin; TP, cisplatin + paclitaxel; PF, cisplatin + fluorouracil; DCR , rate of disease control.

\section{Declarations}

Ethics approval and consent to participate The authors are accountable for all aspects of the work in ensuring that questions related to the accuracy or integrity of any part of the work are appropriately investigated and resolved. All procedures performed in this study involving human participants were in accordance with the Declaration of Helsinki (as revised in 2013). The study was approved by the Institutional Review Board (or Ethics Committee) of the West China Hospital, Sichuan University, Chengdu, China. Informed consent was obtained from all patients.

Consent for publication Not applicable.

Availability of data and material All data generated or analyzed during this study are included in this article.

Competing interests The authors declare that they have no competing interests.

Funding This work was supported by the research and development important project of the Science and Technology Bureau in Sichuan [grant number 2018SZ0188].

Authors' Contributions (I) Conception and design: Liang SM, Leng WB, Jiang D, Liu M, Cao D, Zhu Q, Wang YS, Qiu M;(II) Administrative support: Liang SM, Leng WB, Qiu M; (III) Provision of study materials or patients: Liang SM, Leng WB, Qiu M;(IV) Collection and assembly of data: Liang SM, Leng WB, Jiang D, Liu M, Cao D, Zhu Q, Wang YS, Qiu M; (V) Data analysis and interpretation: Liang SM, Leng WB, Jiang D, Liu M, Cao D, Zhu Q, Wang YS, Qiu M; (VI) Manuscript writing: All authors(VII) ;All authors All authors read and approved the final manuscript

Acknowledgements Not applicable.

\section{References}

1. Bray F, Ferlay J, Soerjomataram I, Siegel R, Torre L, Jemal A. Global cancer statistics 2018: GLOBOCAN estimates of incidence and mortality worldwide for 36 cancers in 185 countries. CA: a cancer journal for clinicians. 2018;68(6):394-424.

2. Kang Y-K, Boku N, Satoh T, Ryu M-H, Chao Y, Kato K, et al. Nivolumab in patients with advanced gastric or gastro-oesophageal junction cancer refractory to, or intolerant of, at least two previous chemotherapy regimens (ONO-4538-12, ATTRACTION-2): a randomised, double-blind, placebo-controlled, phase 3 trial. The Lancet. 2017;390(10111):2461-71.

3. Fuchs CS, Doi T, Jang RW, Muro K, Satoh T, Machado M, et al. Safety and Efficacy of Pembrolizumab Monotherapy in Patients With Previously Treated Advanced Gastric and Gastroesophageal Junction Cancer: Phase 2 Clinical KEYNOTE-059 Trial. JAMA Oncol. 2018;4(5):e180013.

4. Shitara K, Özgüroğlu M, Bang YJ, Di Bartolomeo M, Mandalà M, Ryu MH, et al. Pembrolizumab versus paclitaxel for previously treated, advanced gastric or gastro-oesophageal junction cancer (KEYNOTE-061): a randomised, open-label, controlled, phase 3 trial. Lancet. 2018;392(10142):123-33.

5. Boku N, Ryu MH, Kato K, Chung HC, Minashi K, Lee KW, et al. Safety and efficacy of nivolumab in combination with S-1/capecitabine plus oxaliplatin in patients with previously untreated, unresectable, advanced, or recurrent gastric/gastroesophageal junction cancer: interim results of a randomized, phase II trial (ATTRACTION-4). Ann Oncol. 2019;30(2):250-8.

6. Moehler M, Shitara K, Garrido M, Salman P, Shen L, Wyrwicz L, et al. LBA6_PR Nivolumab (nivo) plus chemotherapy (chemo) versus chemo as first-line (1L) treatment for advanced gastric cancer/gastroesophageal junction cancer (GC/GEJC)/esophageal adenocarcinoma (EAC): First results of the CheckMate 649 study. Annals of Oncology. 2020;31.

7. Shitara K, Van Cutsem E, Bang YJ, Fuchs C, Wyrwicz L, Lee KW, et al. Efficacy and Safety of Pembrolizumab or Pembrolizumab Plus Chemotherapy vs Chemotherapy Alone for Patients With First-line, Advanced Gastric Cancer: The KEYNOTE-062 Phase 3 Randomized Clinical Trial. JAMA Oncol. 2020;6(10):1571-80.

8. Cancer Genome Atlas Research N. Comprehensive molecular characterization of gastric adenocarcinoma. Nature. 2014;513(7517):202-9.

9. Panda A, Mehnert JM, Hirshfield KM, Riedlinger G, Damare S, Saunders T, et al. Immune Activation and Benefit From Avelumab in EBV-Positive Gastric Cancer. J Natl Cancer Inst. 2018;110(3):316-20.

10. Kim ST, Cristescu R, Bass AJ, Kim KM, Odegaard JI, Kim K, et al. Comprehensive molecular characterization of clinical responses to PD-1 inhibition in metastatic gastric cancer. Nat Med. 2018;24(9):1449-58.

11. Mishima S, Kawazoe A, Nakamura Y, Sasaki A, Kotani D, Kuboki Y, et al. Clinicopathological and molecular features of responders to nivolumab for patients with advanced gastric cancer. J Immunother Cancer. 2019;7(1):24. 
12. Wang F, Wei XL, Wang FH, Xu N, Shen L, Dai GH, et al. Safety, efficacy and tumor mutational burden as a biomarker of overall survival benefit in chemorefractory gastric cancer treated with toripalimab, a PD-1 antibody in phase Ib/II clinical trial NCT02915432. Ann Oncol. 2019;30(9):1479-86.

13. Qiu M-Z, He C-Y, Yang D-J, Zhou D-L, Zhao B-W, Wang X-J, et al. Observational cohort study of clinical outcome in Epstein-Barr virus associated gastric cancer patients. Therapeutic Advances in Medical Oncology. 2020;12.

14. Xie T, Liu YQ, Zhang ZN, Zhang XT, Gong JF, Qi CS, et al. Positive Status of Epstein-Barr Virus as a Biomarker for Gastric Cancer Immunotherapy: A Prospective Observational Study. Journal of Immunotherapy. 2020;43(4):139-44.

15. Corallo S, Fucà G, Morano F, Salati M, Spallanzani A, Gloghini A, et al. Clinical Behavior and Treatment Response of Epstein-Barr Virus-Positive Metastatic Gastric Cancer: Implications for the Development of Future Trials. Oncologist. 2020;25(9):780-6.

16. Yu Y, Zeng D, Ou Q, Liu S, Li A, Chen Y, et al. Association of Survival and Immune-Related Biomarkers With Immunotherapy in Patients With Non-Small Cell Lung Cancer: A Meta-analysis and Individual Patient-Level Analysis. JAMA Netw Open. 2019;2(7):e196879.

17. Muro K, Chung HC, Shankaran V, Geva R, Catenacci D, Gupta S, et al. Pembrolizumab for patients with PD-L1-positive advanced gastric cancer (KEYNOTE012): a multicentre, open-label, phase $1 \mathrm{~b}$ trial. Lancet Oncol. 2016;17(6):717-26.

18. Lu Z, Peng Z, Liu C, Wang Z, Wang Y, Jiao X, et al. Current Status and Future Perspective of Immunotherapy in Gastrointestinal Cancers. The Innovation. 2020;1(2).

19. Kang BW, Chau I. Current status and future potential of predictive biomarkers for immune checkpoint inhibitors in gastric cancer. ESMO Open. 2020;5(4).

20. Kim J, Kim B, Kang SY, Heo YJ, Park SH, Kim ST, et al. Tumor Mutational Burden Determined by Panel Sequencing Predicts Survival After Immunotherapy in Patients With Advanced Gastric Cancer. Front Oncol. 2020;10:314.

21. Akin Telli T, Bregni G, Camera S, Deleporte A, Hendlisz A, Sclafani F. PD-1 and PD-L1 inhibitors in oesophago-gastric cancers. Cancer Lett. 2020;469:14250.

22. Ribas A. Adaptive Immune Resistance: How Cancer Protects from Immune Attack. Cancer Discov. 2015;5(9):915-9.

23. Derks S, de Klerk LK, Xu X, Fleitas T, Liu KX, Liu Y, et al. Characterizing diversity in the tumor-immune microenvironment of distinct subclasses of gastroesophageal adenocarcinomas. Ann Oncol. 2020;31(8):1011-20.

24. Binnewies M, Roberts EW, Kersten K, Chan V, Fearon DF, Merad M, et al. Understanding the tumor immune microenvironment (TIME) for effective therapy. Nat Med. 2018;24(5):541-50.

25. Sanmamed MF, Chen L. A Paradigm Shift in Cancer Immunotherapy: From Enhancement to Normalization. Cell. 2018;175(2):313-26.

26. Zhang Y, Chen L. Classification of Advanced Human Cancers Based on Tumor Immunity in the MicroEnvironment (TIME) for Cancer Immunotherapy. JAMA Oncol. 2016;2(11):1403-4.

27. Ma J, Li J, Hao Y, Nie Y, Li Z, Qian M, et al. Differentiated tumor immune microenvironment of Epstein-Barr virus-associated and negative gastric cancer: implication in prognosis and immunotherapy. Oncotarget. 2017;8(40):67094-103.

28. Ott PA, Bang YJ, Piha-Paul SA, Razak ARA, Bennouna J, Soria JC, et al. T-Cell-Inflamed Gene-Expression Profile, Programmed Death Ligand 1 Expression, and Tumor Mutational Burden Predict Efficacy in Patients Treated With Pembrolizumab Across 20 Cancers: KEYNOTE-028. J Clin Oncol. 2019;37(4):31827.

\section{Figures}




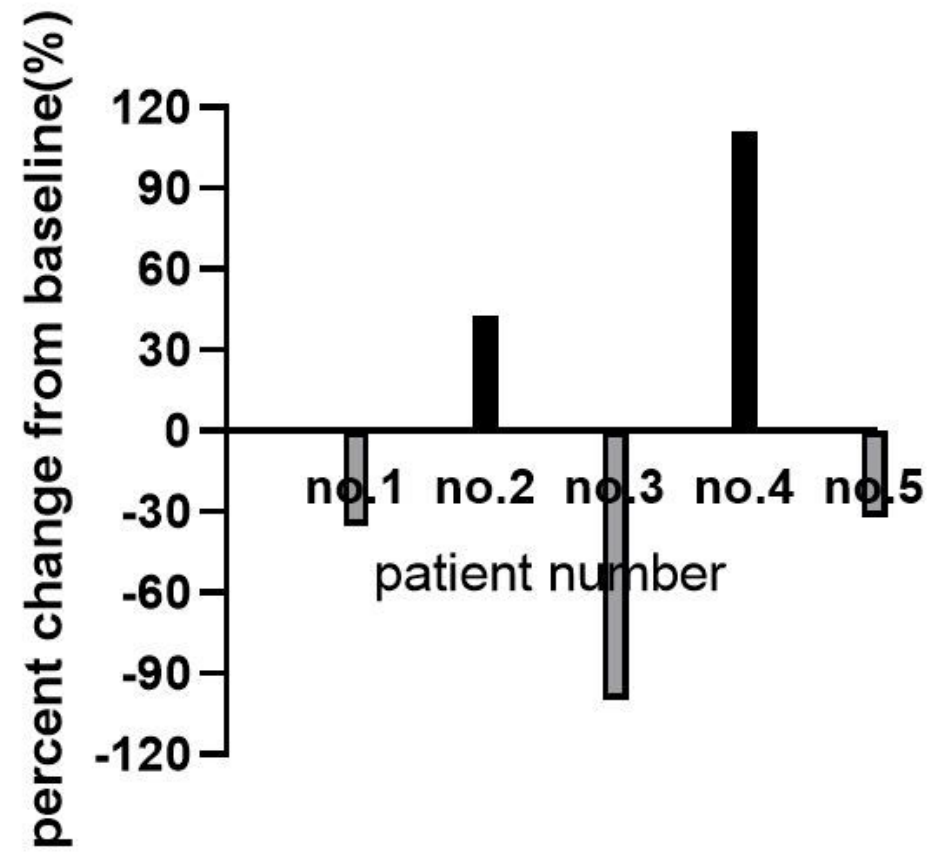

Figure 1

Waterfall Plot of Response to ICls in the patients of our center. 